\title{
Serum $S 100 \beta$ as a predictor of severity and outcomes for mixed subtype acute ischaemic stroke
}

\author{
Siwat Sakdejayont ${ }^{1}$, MD, Nannapat $\underline{\text { Pruphetkaew }}^{2}$, MSc, Pensri $\underline{\text { Chongphattararot }}^{1}$, BSc, \\ Paveena Nanphan ${ }^{1}$, Bsc, Pornchai Sathirapanya ${ }^{1}$, MD
}

INTRODUCTION Serum $\mathrm{S} 100 \beta$ levels are mostly used for predicting outcomes of large-vessel stroke. Its application to mixed subtypes of acute ischaemic stroke (AIS) has been limited.

METHODS Patients with mixed subtypes of AIS who were aged over 18 years and presented within 24 hours of stroke onset were consecutively enrolled. Serum $S 100 \beta$ levels at presentation $\left(S 100 \beta_{b}\right)$ and 72 hours $\left(S 100 \beta_{72 h r s}\right)$, and corresponding National Institutes of Health Stroke Scale (NIHSS $_{b}$ and NIHSS $_{72 h r s}$, respectively) scores were assessed. Stroke outcomes were evaluated using the modified Rankin Scale $(\mathrm{mRs})$ at 30 days $\left(\mathrm{mRs}_{30}\right)$ and 90 days $\left(\mathrm{mRs}_{90}\right)$. Correlations between $S 100 \beta_{b}$ and $S 100 \beta_{72 h r s}$, as well as differences between the two $(\triangle S 100 \beta)$ and the corresponding NIHSS, $\mathrm{mRs}_{30}$ and $\mathrm{mRs}_{90}$ scores, were evaluated $(p<0.05)$.

RESULTS 35 patients were eligible for analysis. On univariate analysis, stroke outcomes had a significant association with $S 100 \beta_{b}, S 100 \beta_{72 h r s}, N_{H S S}, N_{b} S_{72 h r s}$ and $\triangle S 100 \beta$. Both $S 100 \beta_{b}$ and $S 100 \beta_{72 h r s}$ correlated with corresponding NIHSS values $\left(\rho_{b}=0.51, p<0.001 ; \rho_{72 h r s}=0.74, p<0.001\right), \mathrm{mRs}_{30}\left(\rho_{b}=0.58, p<0.001 ; \rho_{72 h r s}=0.72, p<0.001\right)$ and $\mathrm{mRs}$ o0 $\left(\rho_{b}=0.51, p=0.002 ; \rho_{72 h r s}=0.68, p<0.001\right)$. Correlations existed between $\Delta S 100 \beta$ and $\mathrm{mRs}_{30}(\rho=0.74, p<0.001)$ and $\mathrm{mRs}_{90}(\rho=0.71, \mathrm{p}<0.001)$. Practical cut-off points for unfavourable outcomes (mRs 3-6) were S100 $\beta_{72 \mathrm{hrs}}>0.288 \mu \mathrm{g} / \mathrm{L}$ (sensitivity $92.3 \%$, specificity $86.4 \%$ ) and $\Delta S 100 \beta>0.125 \mu \mathrm{g} / \mathrm{L}$ (sensitivity $100 \%$, specificity $81.8 \%$ ).

CONCLUSION High serum $S 100 \beta$ is associated with unfavourable outcomes for mixed subtype AIS. Cut-off values of $\mathrm{S} 100 \beta_{72 \mathrm{hrs}}$ and $\triangle \mathrm{S} 100 \beta$ were optimal for predicting unfavourable stroke outcomes.

Keywords: acute ischaemic stroke, outcome, serum S100ß

\section{INTRODUCTION}

Various neural-specific biomarkers are released into the serum and cerebrospinal fluid during neural tissue injuries. Both direct and indirect cerebral damage (e.g. cerebral trauma, cardiopulmonary arrest, postoperative neurovascular complications and acute ischaemic stroke [AIS]) lead to the release of these biomarkers. ${ }^{(1-5)}$ Thus, assessment of the presence and magnitude of such biomarkers during neurovascular insults may facilitate not only the diagnosis but also the prediction of the complications and final outcomes of the neural injury. ${ }^{(1,2,6)}$ A recent study found that each biomarker has a specific cellular origin within the cerebral tissue (e.g. neuron-specific enolase [NSE] is released from neurons; serum S100 $\beta$ is released from astrocytes and Schwann cells; Tau protein is from neurons; metalloproteinase- 9 is released from vascular endothelium; and ubiquitin $\mathrm{C}$ terminal hydroxylase-L1 is released from neurons). ${ }^{(3)}$ Therefore, several biomarkers associated with specific cerebral cellular damage have been under extensive investigation to identify novel biomarkers that could serve these purposes. ${ }^{(7)}$ Recently, among many stroke-related biomarkers that were widely evaluated for their suitability for clinical deployment, S100 $\beta$ and NSE were the two most broadly attributed in many clinical studies for their clinical usefulness. ${ }^{(2,3,6,8-12)}$ Because of the greater clinically correlated response of $\mathrm{S} 100 \beta$ and absence of interference by the body's clearance systems, there has been much interest in proving its clinical applicability. ${ }^{(3)}$
The $\mathrm{S} 100 \beta$ protein is an acidic, calcium-binding, neuralspecific biomarker. It is a $\sim 21-k D a$ protein with a homodimeric structure (double helix loop) that is encoded on the long arm of chromosome 21 (21q22.3). ${ }^{(2,13)}$ As S100 $\beta$ has been used as a target of immunologically specific staining techniques to demonstrate astrocytes and melanocytes, it is a potentially promising biomarker for damage to astroglial cells as well as melanocyte proliferation. ${ }^{(3,7)}$ Furthermore, a study based on experimental induction of endothelial injury has suggested that $\mathrm{S} 100 \beta$ could be a biomarker for blood-brain barrier disruption. ${ }^{(14)}$

Several studies have reported a significant elevation of serial S100 $\beta$ levels following AIS, in particular among patients with large-vessel or cortical brain infarction. The elevation of S100 $\beta$, both initially and subsequently at the appropriate time points, significantly correlated with not only the extent of cerebral infarction, as reflected by stroke severity, but also the associated complications and neurological outcomes. ${ }^{(8-12,15,16)}$

Earlier studies have reported that $\mathrm{S} 100 \beta$ was first detected in the serum of patients with AIS at 6-12 hours and reached peak levels at 72 hours after the onset of AIS. ${ }^{(12,17,18)}$ It followed firstorder kinetics, with a biological half-life of $25.3 \pm 5.1$ minutes, without effect from a moderately decreased glomerular filtration rate. ${ }^{(5)}$ Nevertheless, due to the delayed kinetics for the first detection in serum (6-12 hours), S100 $\beta$ is not an ideal biomarker for AIS diagnosis. ${ }^{(12,18)}$

${ }^{1}$ Division of Neurology, Department of Internal Medicine, ${ }^{2}$ Epidemiology Unit, Faculty of Medicine, Prince of Songkla University, Hat Yai, Songkhla, Thailand Correspondence: Dr Pornchai Sathirapanya, Associate Professor, Division of Neurology, Department of Internal Medicine, Faculty of Medicine, Prince of Songkla University, Hat Yai, Songkhla 90110, Thailand. sporncha@medicine.psu.ac.th 
To the best of our knowledge, there has been limited evaluation of the clinical usefulness of $\mathrm{S} 100 \beta$ in mixed cortical (or large-vessel occlusive stroke [LVS]) and subcortical (or small-vessel occlusive stroke [SVS]) AIS. Therefore, in the present study, we aimed to: (a) demonstrate the significance of the correlations between the initial and subsequent (at 72 hours after AIS onset) S100 $\beta$ levels with the severity and outcomes of patients with mixed subtype AIS; and (b) determine the optimal cut-off points of S100 $\beta$ levels at each time point for predicting unfavourable outcomes in patients with mixed subtype AIS. We intended to prove the clinical applicability of $S 100 \beta$ for predicting the outcomes of patients with mixed cortical and subcortical ischaemic strokes.

\section{METHODS}

We prospectively enrolled all patients aged over 18 years who presented to Songklanagarind Hospital, Faculty of Medicine, Prince of Songkla University, Songkhla, Thailand, from January 2013 to September 2013 with newly diagnosed AIS within 24 hours of the onset of symptoms. The diagnosis of AIS was based on the patient's clinical presentation and confirmed by either computed tomography or magnetic resonance imaging of the brain. Patients with a previous history of neurological illness, existing neurological disorder or residual neurological disability from any cause (e.g. stroke, cerebral neoplasm, intracranial infection or traumatic cerebral injury) were excluded. To achieve statistically significant power, a sample size of 36 was necessary based on the equation:

$$
\therefore n_{1}=\frac{\left[Z_{1-\alpha / 2} \sqrt{p(1-p)(1+1 / r)}+Z_{\beta} \sqrt{\begin{array}{l}
p 1(1-p 1)+ \\
p 2(1-p 2) / r
\end{array}}\right]^{2}}{\Delta^{2}}
$$

where proportions of positive outcomes among the exposed group $(p 1)=0.73$ and proportions of positives outcome among the nonexposed group $(\mathrm{p} 2)=0.17, \alpha=0.05$, power $=0.8$, and $r=1$.

Patients' demographic data and cardiovascular risk factors were collected during stroke risk screening. Presenting stroke severity was graded using the National Institutes of Health Stroke Scale score (NIHSS) immediately upon the patient's arrival at our centre, as the baseline severity scale $\left(\mathrm{NIHSS}_{\mathrm{b}}\right)$. An emergency cranial imaging study, in most cases computed tomography of the brain, was performed to confirm the diagnosis and classify the subtype of AIS, in combination with the clinical stroke syndrome. All brain images were subsequently reviewed and confirmed by a clinically blinded neuroradiologist. The first blood sample for the baseline assay of $\mathrm{S} 100 \beta\left(\mathrm{S} 100 \beta_{\mathrm{b}}\right)$ was collected prior to starting treatment.

The primary AIS treatment consisted of one or a combination of the following: intravenous thrombolysis, endovascular thrombectomy and antithrombotic agents. The second S100 $\beta$ assay was performed at 72 hours after the onset of stroke symptoms $\left(\mathrm{S} 100 \beta_{72 \mathrm{hrs}}\right)$, together with a re-evaluation of stroke severity using NIHSS $\left(\mathrm{NIHSS}_{72 \mathrm{hrs}}\right.$ ). Stroke-related complications (e.g. haemorrhagic transformation and malignant cerebral oedema necessitating decompressive craniectomy) were monitored. Stroke outcomes were assessed using the modified Rankin Scale (mRs) at 30 days $\left(\mathrm{mRs}_{30}\right)$ and 90 days $\left(\mathrm{mRs}_{90}\right)$ after AIS onset. mRs $\leq 2$ was considered as favourable, while mRs 3-6 was unfavourable.
Measurement of serum S100 $\beta$ levels was performed using a commercial kit (Elecsys S100; Roche Diagnostics, Indianapolis, IN, USA) that was composed of a two-site monoclonal S100-specific antibody to form a sandwich complex on a fully automated system (Elecsys 2010, Modular Analytics E170; Roche Diagnostics-Hitachi High-Technologies Corporation, Tokyo, Japan). The range of measurement was $0.015-30.0 \mu \mathrm{g} / \mathrm{L}$. Values were evaluated within an intra-assay coefficient of variation in the range of $1.28 \%-2.32 \%$.

Descriptive statistics were shown as number and percentage, mean \pm standard deviation or median (interquartile range [IQR]). Fisher's exact test and chi-square test were used to analyse categorical variables, whereas Student's $t$-test and Wilcoxon ranksum test were used to analyse continuous variables for statistical significance. A p-value $<0.05$ was considered to be statistically significant. Continuous variables were assessed using the ShapiroWilk test for normality. Correlations between $\mathrm{S} 100 \beta_{\mathrm{b}}$ and $\mathrm{S} 100 \beta_{72 \mathrm{hrs}}$ and the corresponding $\mathrm{NIHSS}_{\mathrm{b}}$ and $\mathrm{NIHSS}_{72 \mathrm{hrs}}$ values as well as correlations between each $\mathrm{S} 100 \beta$ level and the difference of the two $\mathrm{S} 100 \beta$ measurements $(\Delta \mathrm{S} 100 \beta)$ with $\mathrm{mRs}_{30}$ and $\mathrm{mRs}_{90}$ were evaluated using Spearman's correlation. The optimal cut-off points of $S 100 \beta_{b^{\prime}} S 100 \beta_{72 h r s}$ and $\Delta S 100 \beta$ levels to predict final patient outcomes and mortality were obtained using receiver operating characteristic (ROC) curves. The sensitivity, specificity, positive predictive value and negative predictive value of each $\mathrm{S} 100 \beta$ and $\Delta$ S100 $\beta$ cut-off point obtained were also calculated.

The complete study protocol was reviewed and approved by the ethics committee of the Faculty of Medicine, Prince of Songkla University, Songkhla, Thailand. We strictly followed the regulations of the 1964 Declaration of Helsinki and its later amendments while performing the study. All identifiable personal information of enrolled patients was made completely anonymous.

\section{RESULTS}

In total, 35 out of 44 enrolled patients with AIS were eligible for final analysis after excluding six patients who met the exclusion criteria and three patients who had incomplete follow-up. There were 26 men and nine women with an overall mean age of $65.3 \pm 10.7$ (range $43-81$ ) years (Table I). The men were slightly older than the women.

Smoking, hypertension and hyperlipidaemia were sequentially the three most common cardiovascular risk factors among our patients. The total number of SVSs was 17 (48.6\%), involving 15 SVSs and two other undetermined subtypes that were finally classified as SVSs. The median NIHSS bas 10.0 (IQR 6.0-19.0), while the median NIHSS $_{72 \text { hrs }}$ was 6.0 (IQR 2.5-16.0). The $\mathrm{S} 100 \beta_{\mathrm{b}}$ assessments were available at a mean time of $10.6 \pm 7.3$ (range 2.0-24.0) hours after stroke onset, while the $S 100 \beta_{72 \text { hrs }}$ assessments were performed at 72 hours after stroke onset.

For primary stroke treatment, 21 patients received conventional therapy in the form of antiplatelet or anticoagulant therapy, while ten patients received intravenous thrombolysis and one patient underwent endovascular thrombectomy. Primary stroke treatment was withheld for three patients - two patients had haemorrhagic transformation and one patient had massive cerebral oedema 
Table I. Univariate logistic regression analysis of clinical variables and stroke outcomes at 30 days and 90 days.

\begin{tabular}{|c|c|c|c|c|}
\hline \multirow[t]{2}{*}{ Variable } & \multicolumn{3}{|c|}{ No. (\%) } & \multirow[t]{2}{*}{ p-value } \\
\hline & Total $(n=35)$ & $m R s 0-2 *(n=22)$ & $m R s 3-6 *(n=13)$ & \\
\hline Gender & & & & $>0.999$ \\
\hline Male & $26(74.3)$ & $16(72.7)$ & $10(76.9)$ & \\
\hline Female & $9(25.7)$ & $6(27.3)$ & $3(23.1)$ & \\
\hline $\mathrm{Age}^{\dagger}(\mathrm{yr})$ & $65.3 \pm 10.7$ & $63.8 \pm 10.2$ & $67.8 \pm 11.5$ & 0.289 \\
\hline Male & $66.5 \pm 11.1$ & & & \\
\hline Female & $61.6 \pm 8.9$ & & & \\
\hline \multicolumn{5}{|l|}{ Risk factor } \\
\hline Smoking & $20(57.1)$ & $12(54.5)$ & $8(61.5)$ & 0.960 \\
\hline Hypertension & $18(51.4)$ & $12(54.5)$ & $6(46.2)$ & 0.897 \\
\hline Dyslipidaemia & $10(28.6)$ & $8(36.4)$ & $2(15.4)$ & 0.259 \\
\hline Diabetes mellitus & $7(20.0)$ & $2(9.1)$ & $5(38.5)$ & 0.075 \\
\hline Atrial fibrillation & $6(17.1)$ & $2(9.1)$ & $4(30.8)$ & 0.166 \\
\hline Coronary artery disease & $4(11.4)$ & $1(4.5)$ & $3(23.1)$ & 0.134 \\
\hline \multicolumn{5}{|l|}{ TOAST classification } \\
\hline Large vessel & $13(37.1)$ & $5(22.7)$ & $8(61.5)$ & \\
\hline Small vessel & $15(42.9)$ & $14(63.6)$ & $1(7.7)$ & \\
\hline Cardioembolic & $5(14.3)$ & $1(4.5)$ & $4(30.8)$ & \\
\hline Others (determined) & $0(0)$ & $0(0)$ & $0(0)$ & \\
\hline Others (undetermined) & $2(5.7)$ & $2(9.1)$ & $0(0)$ & \\
\hline \multicolumn{5}{|l|}{ NIHSS $^{\ddagger}$} \\
\hline At baseline & $10.0(6.0-19.0)$ & $7.0(4.2-9.8)$ & $20.0(19.0-21.0)$ & $<0.001^{\S}$ \\
\hline At $72 \mathrm{hr}$ & $6.0(2.5-16.0)$ & $3.0(2.0-4.8)$ & $18.0(16.0-28.0)$ & $<0.001^{\S}$ \\
\hline \multicolumn{5}{|l|}{$S 100 \beta^{\ddagger}(\mu \mathrm{g} / \mathrm{L})$} \\
\hline At baseline & $0.1(0.1-0.2)$ & $0.1(0.1-0.1)$ & $0.2(0.1-0.2)$ & $<0.001^{\S}$ \\
\hline At $72 \mathrm{hr}$ & $0.2(0.1-0.7)$ & $0.1(0.1-0.2)$ & $1.0(0.5-4.1)$ & $<0.001^{\S}$ \\
\hline Difference from baseline & $0.1(0.0-0.5)$ & $0(0.0-0.1)$ & $0.9(0.3-3.8)$ & $<0.001^{\S}$ \\
\hline \multicolumn{5}{|l|}{ Treatment } \\
\hline Conventional therapy & $21(60.0)$ & $16(72.7)$ & $5(38.5)$ & \\
\hline Intravenous thrombolysis & $10(28.6)$ & $5(22.7)$ & $5(38.5)$ & \\
\hline Endovascular thrombectomy & $1(2.9)$ & $0(0.0)$ & $1(7.7)$ & \\
\hline Haemorrhagic transformation & $5(14.3)$ & $1(4.5)$ & $4(30.8)$ & \\
\hline Decompression craniotomy & $2(5.7)$ & $0(0.0)$ & $2(15.4)$ & \\
\hline
\end{tabular}

*mRs score at 30 days and 90 days after stroke were identical ( $m R s ~ 0-2=$ favourable outcome, $m R s ~ 3-6=$ unfavourable outcome). + Data presented as mean \pm standard deviation. $\neq$ Data presented as median (interquartile range). $\$ p<0.05$ was statistically significant using Mann-Whitney $U$ test. mRs: modified Rankin Scale score at 30 days and 90 days after stroke; NIHSS: National Institutes of Health Stroke Scale score; S100ß: serum S100ß;TOAST classification: Trial of ORG 10172 in acute stroke treatment classification

initially. The final stroke outcomes evaluated using $\mathrm{mRs}_{30}$ and $\mathrm{mRs}_{90}$, which were categorised as favourable (mRs $\left.\leq 2\right)$ or unfavourable (mRs 3-6), were identical for the categorical outcomes (favourable or unfavourable) at both time points for each patient. Five patients had complications of haemorrhagic transformation, and two patients developed malignant brain oedema for which decompression craniotomy was indicated. Eventually, 22 patients had a favourable outcome, while 13 patients had an unfavourable outcome. Six of the patients died of direct stroke-related severity and complications.

$\mathrm{S} 100 \beta_{\mathrm{b}^{\prime}}, \mathrm{S} 100 \beta_{72 \mathrm{hrs}^{\prime}} \Delta \mathrm{S} 100 \beta, \mathrm{NIHSS}_{\mathrm{b}}$ and $\mathrm{NIHSS}_{72 \mathrm{hrs}}$ were significantly associated with categorical stroke outcomes on univariate logistic regression analysis (Table I). Further evaluation of $\mathrm{S} 100 \beta_{\mathrm{b}}$ showed positive correlations with $\mathrm{NIHSS}_{\mathrm{b}}(\rho=0.51$, $\mathrm{p}<0.001), \mathrm{mRs}_{30}(\rho=0.58, \mathrm{p}<0.001)$ and $\mathrm{mRs}_{90}(\rho=0.51, \mathrm{p}=0.002)$ using Spearman's correlation (Fig. 1). $S 100 \beta_{72 h r s}$ also had positive correlations with NIHSS ${ }_{72 \text { hrs }}(\rho=0.74, p<0.001), \mathrm{mRs}_{30}(\rho=0.72$, $\mathrm{p}<0.001)$ and $\mathrm{mRs}_{90}(\rho=0.68, \mathrm{p}<0.001)$ (Fig. 2). The difference between $S 100 \beta_{72 \text { hrs }}$ and $S 100 \beta_{b}$ (i.e. $\Delta S 100 \beta$ ) also showed a positive correlation with $\mathrm{mRs}_{30}(\rho=0.74, \mathrm{p}<0.001)$ and $\mathrm{mRs}_{90}(\rho=0.71$, $\mathrm{p}<0.001$ ) but no significant correlation with the difference between $\mathrm{NIHSS}_{72 \mathrm{hrs}}$ and $\mathrm{NIHSS}_{\mathrm{b}}$ (i.e. $\Delta \mathrm{NIHSS}, \rho=0.07, \mathrm{p}=0.700$ ) (Fig. 3).

The optimal cut-off points for $S 100 \beta_{b^{\prime}} S 100 \beta_{72 \mathrm{hrs}}$ and $\Delta S 100 \beta$ for the prediction of unfavourable stroke outcomes and death, 

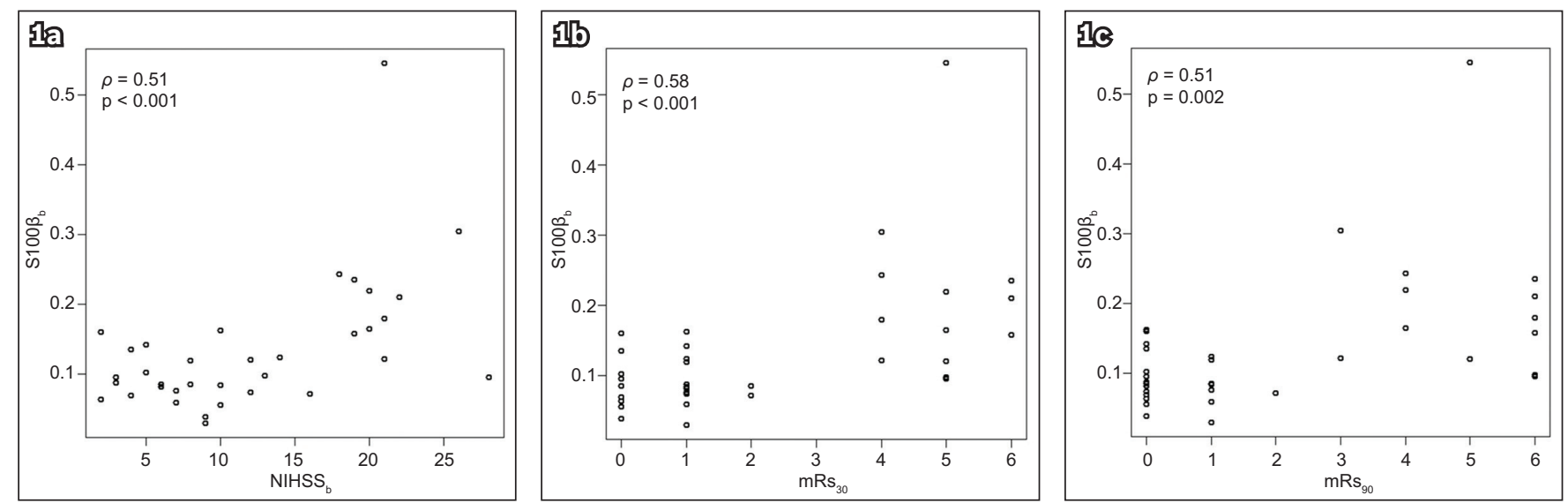

Fig. 1 Charts show the correlation of serum $S 100 \beta$ at baseline with (a) NIHSS score at baseline; (b) mRs score at 30 days after stroke; and (c) mRs score at 90 days after stroke. mRs: modified Rankin Scale; NIHSS : National Institute of Health and Stroke Scale score
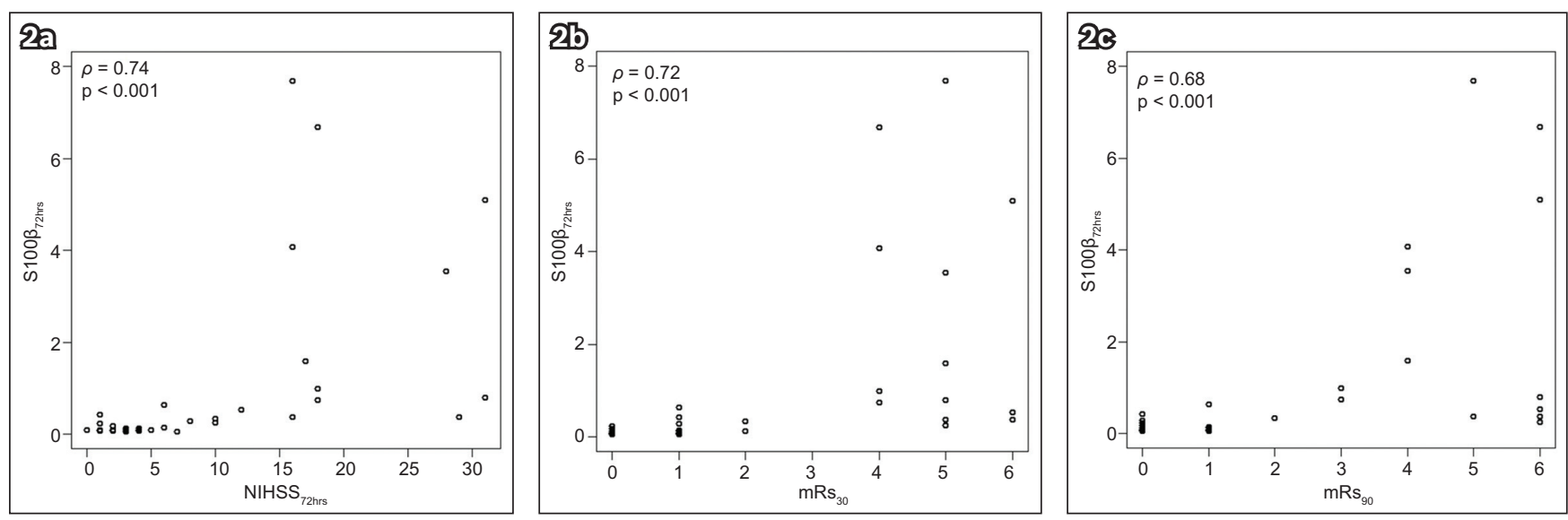

Fig. 2 Charts show correlation of serum $\mathrm{S} 100 \beta$ at 72 hours after stroke onset with (a) NIHSS score at 72 hours after stroke onset; (b) mRs score at 30 days after stroke; and (c) mRs score at 90 days after stroke. mRs: modified Rankin Scale; NIHSS: National Institutes of Health Stroke Scale score
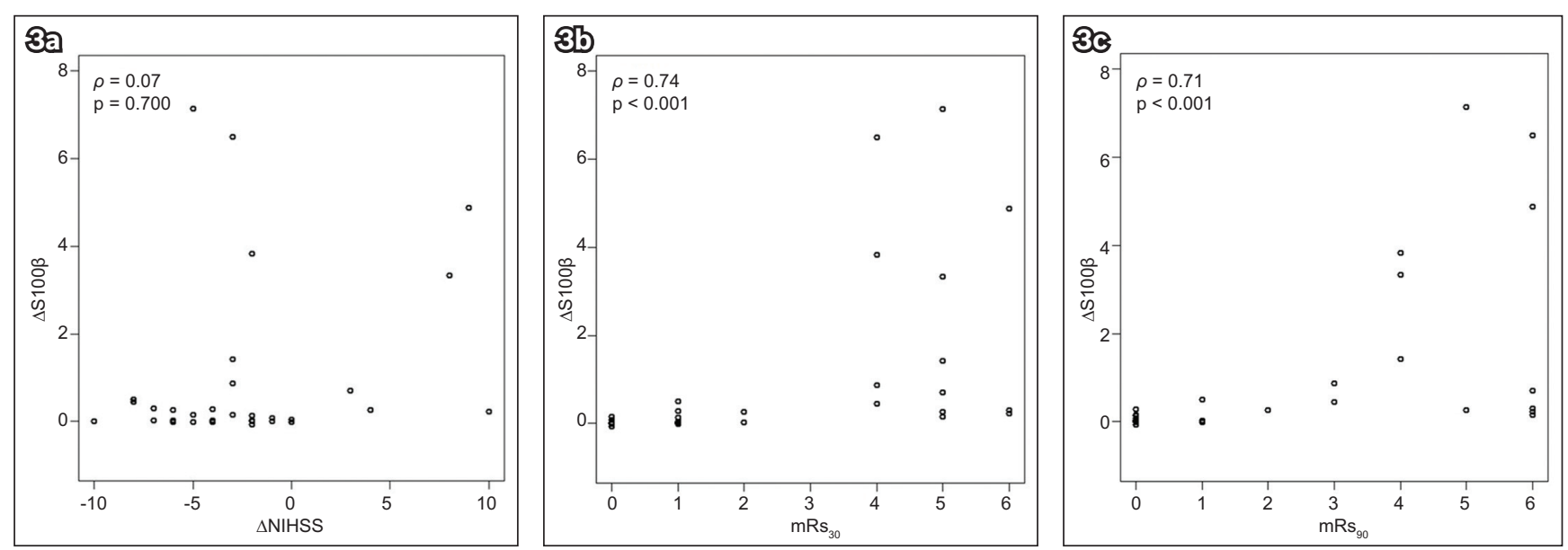

Fig. 3 Charts show correlation of $\triangle S 100 \beta$ with (a) $\Delta$ NIHSS; (b) mRs score at 30 days after stroke; and (c) mRs score at 90 days after stroke. $\Delta$ NIHSS: difference between National Institutes of Health Stroke Scale score at baseline and at 72 hours after stroke onset; $\triangle S 100 \beta$ : difference between serum $\mathrm{S} 100 \beta$ at baseline and at 72 hours after stroke onset; mRs: modified Rankin Scale

as well as sensitivity, specificity, area under the curve, positive predictive value and negative predictive value identified by the ROC curves, are presented in Table II.

\section{DISCUSSION}

Unlike most previous studies that involved large vessel thrombotic and embolic strokes, 17 (48.6\%) of 35 patients in our study were classified as SVS. We found that $\mathrm{S} 100 \beta_{\mathrm{b}^{\prime}}, \mathrm{S} 100 \beta_{72 \mathrm{hrs}} \Delta \mathrm{S} 100 \beta$, $\mathrm{NIHSS}_{\mathrm{b}}$ and NIHSS${ }_{72 \mathrm{hr}}$ had a significant association with final stroke outcomes upon univariate logistic regression analysis. However, statistical significance was not found following multivariate logistic regression analysis of all NIHSS and S100 $\beta$ values. Therefore, $\mathrm{S} 100 \beta_{\mathrm{b}}$ and $\mathrm{S} 100 \beta_{72 \mathrm{hrs}}$ may not be superior to $\mathrm{NIHSS}_{\mathrm{b}}$ and $\mathrm{NIHSS} \mathrm{H}_{72 \mathrm{hrs}}$ for predicting the final functional outcome of patients with stroke. 
Table II. Optimal cut-off points for serum $\mathrm{S100} \beta$ at baseline and at 72 hours after stroke onset for predicting unfavourable stroke outcome $(\mathrm{mRs}>2)$ and death ( $\mathrm{mRs} 6)$ at 30 days and 90 days after stroke.

\begin{tabular}{|c|c|c|c|c|c|c|}
\hline Prediction & S100 $\beta$ level $(\mu \mathrm{g} / \mathrm{L})$ & Sensitivity (\%) & Specificity (\%) & AUC & PPV (\%) & NPV (\%) \\
\hline \multicolumn{7}{|c|}{ Unfavourable outcome $(n=13)$} \\
\hline \multicolumn{7}{|c|}{ All stroke $(n=13)$} \\
\hline$S 100 \beta_{b}$ & 0.096 & 92.3 & 68.2 & 0.80 & 63.0 & 93.0 \\
\hline$S 100 \beta_{72 h r s}$ & 0.288 & 92.3 & 86.4 & 0.89 & 80.0 & 95.0 \\
\hline$\Delta S 100 \beta$ & 0.125 & 100.0 & 81.8 & 0.91 & 76.5 & 100.0 \\
\hline \multicolumn{7}{|c|}{ Large vessel stroke $(n=12)$} \\
\hline$S 100 \beta_{b}$ & 0.158 & 75.0 & 100.0 & 0.88 & 100.0 & 67.0 \\
\hline$S 100 \beta_{72 h r s}$ & 0.745 & 75.0 & 100.0 & 0.88 & 100.0 & 67.0 \\
\hline$\Delta S 100 \beta$ & 0.294 & 83.3 & 83.3 & 0.83 & 90.9 & 71.4 \\
\hline \multicolumn{7}{|c|}{ Small vessel stroke $(n=1)$} \\
\hline$S 100 \beta_{b}$ & 0.098 & 100.0 & 69.0 & 0.84 & 16.0 & 100.0 \\
\hline$S 100 \beta_{72 h r s}$ & 0.242 & 100.0 & 87.0 & 0.94 & 33.0 & 100.0 \\
\hline$\Delta S 100 \beta$ & 0.125 & 100.0 & 81.2 & 0.90 & 25.0 & 100.0 \\
\hline \multicolumn{7}{|l|}{ Death $(n=6)$} \\
\hline \multicolumn{7}{|l|}{ At 30 days } \\
\hline$S 100 \beta_{b}$ & 0.158 & 100.0 & 75.0 & 0.88 & 27.3 & 100.0 \\
\hline$S 100 \beta_{72 h r s}$ & 0.375 & 100.0 & 65.6 & 0.83 & 21.4 & 100.0 \\
\hline \multicolumn{7}{|l|}{ At 90 days } \\
\hline$S 100 \beta_{b}$ & 0.096 & 100.0 & 48.3 & 0.74 & 28.6 & 100.0 \\
\hline$S 100 \beta_{72 h r s}$ & 0.242 & 100.0 & 62.1 & 0.81 & 35.3 & 100.0 \\
\hline
\end{tabular}

$\triangle S 100 \beta$ : difference between serum S100 $\beta$ at baseline and at 72 hours after stroke onset; AUC: area under the curve; mRs: modified Rankin Scale; NPV: negative predictive value; PPV: positive predictive value; $S 100 \beta_{72 \text { hrs }}$ : serum S100 $\beta$ at 72 hours after stroke onset; S100 $\beta_{b}$ : serum S100 $\beta$ at baseline

Several widely reported previous clinical studies regarding the usefulness of $\mathrm{S} 100 \beta$ and other more specific neuronal biomarkers were based on patients with LVS and mostly middle cerebral artery occlusion. Foerch et al ${ }^{(16)}$ reported a correlation between a single $S 100 \beta$ measurement and functional outcome and also brain infarction volume seven days after stroke. They also found that the $S 100 \beta$ level was not influenced by variables such as age, gender, stroke severity, aetiology, size of lesion and risk factors upon multivariate logistic regression analysis. ${ }^{(16)}$ Furthermore, a single-measurement study of $\mathrm{S} 100 \beta$ at 48 hours after stroke onset reported that $\mathrm{S} 100 \beta$ level $\leq 0.37 \mu \mathrm{g} / \mathrm{L}$ could predict an independent functional outcome, with a sensitivity of $87 \%$ and specificity of $78 \%$ for patients with middle cerebral artery infarction. ${ }^{(16)}$ Another study confirmed that a S100 $\beta$ cut-off level $>0.2 \mu \mathrm{g} / \mathrm{L}$ at 48 hours after stroke in patients with AIS was a strong predictor for unfavourable functional outcome at three months after stroke onset, with a sensitivity of $85 \%$ and specificity of $92 \%$. Moreover, a study demonstrated that elevation of S100 $\beta$ as early as six hours after stroke onset was associated with an unfavourable functional outcome. ${ }^{(12)}$ Fassbender et $\mathrm{al}^{(8)}$ showed a correlation between elevated serum S100 $\beta$ levels at ten hours, 24 hours and 72 hours after stroke onset with an infarction volume $>5 \mathrm{~mm}^{3}$ and functional outcome. In their study, the elevation of S100 $\beta$ was found to be significantly higher for cortical infarctions than for subcortical and brain stem infarctions at 24 hours and 72 hours after stroke onset. ${ }^{(8)}$

In our study, the first blood sample for the $\mathrm{S} 100 \beta$ assay was collected within 12 hours and, significantly, a second sample was collected at 72 hours after stroke onset, which was considered the most optimal time point to assess $\mathrm{S} 100 \beta$ levels for estimating the extent of cerebral damage after stroke. This time point for the second S100 $\beta$ measurement was selected based on the findings of a previous experimental animal study and a clinical review of the role of $S 100 \beta$ in AIS. ${ }^{(17,19)}$ Although the level of $S 100 \beta$ in our study was not identical to previous studies, we found a comparable correlation with stroke severity as well as short- and long-term functional outcomes, as evaluated by $\mathrm{mRs}_{30}$ and $\mathrm{mRs}_{90^{\prime}}$ among our patients. Similarly, $\Delta S 100 \beta$, in our study, showed a correlation with $\mathrm{mRs}_{30}$ and $\mathrm{mRs}_{90}$

The cut-off values of $\mathrm{S} 100 \beta_{\mathrm{b}^{\prime}} \mathrm{S} 100 \beta_{72 \mathrm{hrs}}$ and $\Delta \mathrm{S} 100 \beta$ were good predictors of unfavourable outcome for all stroke subtypes in our study. However, as only one patient with SVS who experienced unfavourable outcome was included, our findings vis-à-vis the preciseness and generalisation of the cut-off values for $\mathrm{S} 100 \beta$ to all patients with stroke may be limited. Additionally, the cut-off values found for $S 100 \beta_{72 \text { hrs }}$ and $\Delta S 100 \beta$ were notably more appropriate than $S 100 \beta_{b}$ in predicting an unfavourable functional outcome. Therefore, similar to previous studies, $(8,17,19)$ we also propose that $\mathrm{S} 100 \beta$ measurement is appropriate for the prediction of unfavourable stroke outcome among patients with LVS, while its usefulness in patients with SVS remains as yet undetermined. Furthermore, a single measurement at 72 hours, or $\mathrm{S} 100 \beta_{72 \mathrm{hrs}}$, has the highest specificity for predicting an unfavourable outcome of AIS.

Most studies have recognised the clinical usefulness of assessments of $S 100 \beta$ levels for estimating the extent of cerebral 
ischaemic damage that is reflected in infarction volume, monitoring patients' response to treatment and their subsequent functional outcome, as assessed by mRs. ${ }^{(6,8,12,15,16,18,20)}$ The $\mathrm{S} 100 \beta$ level can be detected in the serum as early as 6-12 hours after stroke onset and, in most patients, reaches its peak 24 hours or more after stroke. ${ }^{(18)}$ However, some other studies have reported the $\mathrm{S} 100 \beta$ peak at $48-72$ hours after stroke onset, ${ }^{(8,9,12,17)}$ suggesting that there is as yet no consensus over the optimal time points or number of $\mathrm{S} 100 \beta$ sample assays to be rationally performed for patients with stroke. On the contrary, certain studies have suggested that cerebrospinal fluid might be a more appropriate source for S100 $\beta$ and NSE assessments to predict stroke severity since, with cerebrospinal fluid measurements, there is no interference from other potential extracranial sources of $\mathrm{S} 100 \beta$ such as adipose tissue, melanocytes, T-lymphocytes, skin or skeletal muscles. ${ }^{(2,11)}$ In view of these earlier findings, physicians should exercise caution when using S100 $\beta$ and NSE measurements for evaluating patients with stroke unless such potential extracranial sources are carefully excluded.

Other biomarkers for the prediction of stroke outcome have also been investigated. Jauch et al, in the National Institute of Neurological Disorders and Stroke Recombinant Tissue Plasminogen Activator Stroke Study, demonstrated a positive association between the peak 24-hour level of myelin basic proteins, NSE, S100 $\beta$ and NIHSS at presentation, as well as a worse outcome at three months after stroke among patients with a higher difference of $\mathrm{S} 100 \beta$ and myelin basic proteins levels between 24 hours and two hours after initiation of intravenous thrombolysis treatment. ${ }^{(6)}$ Although NSE followed the same kinetic pattern as S100 $\beta$, it showed no correlation with infarction volume and functional outcomes, ${ }^{(8)}$ suggesting that NSE has no clinical efficacy for the prediction of outcome of AIS.

A difference in the elevation of biomarkers among patients with LVS and SVS has not been clearly elucidated.(2) A study on glial tissue-specific proteins suggested that glial fibrillary acidic protein was more sensitive for estimating brain damage in patients with SVS, whereas S100 $\beta$ was more suitable for patients with LVS. ${ }^{(11)}$

The present study, which was conducted in Thailand, demonstrated that $\mathrm{S} 100 \beta$ had a high specificity for predicting unfavourable outcomes of LVS in an Asian population. Differences in ethnicity have no influence on the $S 100 \beta$ measurements. Further clinical investigations enrolling a larger sample size of various stroke subtypes, with particular emphasis on patients with SVS, are warranted to confirm the true clinical usefulness of $\mathrm{S} 100 \beta$ as a broad stroke biomarker. The optimal number of assessments required and the appropriate time points at which to perform these S100 $\beta$ assessments also need to be specified to standardise practices and to gain a better understanding of the diagnostic and prognostic value of S100 $\beta$ measurements among patients with stroke.

\section{ACKNOWLEDGEMENT}

The study was supported by the Research Fund of the Faculty of Medicine, Prince of Songkla University, Songkhla, Thailand.

\section{REFERENCES}

1. Laskowitz DT, Kasner SE, Saver J, Remmel KS, Jauch EC; BRAIN Study Group. Clinical usefulness of a biomarker-based diagnostic test for acute stroke: the Biomarker Rapid Assessment in Ischemic Injury (BRAIN) study. Stroke 2009; 40:77-85.

2. Persson L, Hårdemark HG, Gustafsson J, et al. S-100 protein and neuron-specific enolase in cerebrospinal fluid and serum: markers of cell damage in human central nervous system. Stroke 1987; 18:911-8.

3. Cata JP, Abdelmalak B, Farag E. Neurological biomarkers in the perioperative period. Br J Anaesth 2011; 107:844-58.

4. Jönsson $\mathrm{H}$, Johnsson $\mathrm{P}$, Alling $\mathrm{C}$, et al. S100 beta after coronary artery surgery: release pattern, source of contamination, and relation to neuropsychological outcome. Ann Thorac Surg 1999; 68:2202-8.

5. Jönsson $\mathrm{H}$, Johnsson $\mathrm{P}$, Höglund $\mathrm{P}$, Alling $\mathrm{C}$, Blomquist $\mathrm{S}$. Elimination of $\mathrm{S} 100 \mathrm{~B}$ and renal function after cardiac surgery. J Cardiothorac Vasc Anesth 2000; 14:698-701.

6. Jauch EC, Lindsell C, Broderick J, et al; NINDS rt-PA Stroke Study Group. Association of serial biochemical markers with acute ischemic stroke: the National Institute of Neurological Disorders and Stroke recombinant tissue plasminogen activator Stroke Study. Stroke 2006; 37:2508-13.

7. Anderson L. Candidate-based proteomics in the search for biomarkers of cardiovascular disease. J Physiol 2005; 563 (Pt 1):23-60.

8. Fassbender K, Schmidt R, Schreiner A, et al. Leakage of brain-originated proteins in peripheral blood: temporal profile and diagnostic value in early ischemic stroke. J Neurol Sci 1997; 148:101-5.

9. Büttner T, Weyers S, Postert T, Sprengelmeyer R, Kuhn W. S-100 protein: serum marker of focal brain damage after ischemic territorial MCA infarction. Stroke 1997; 28:1961-5.

10. Missler U, Wiesmann M, Friedrich C, Kaps M. S-100 protein and neuron-specific enolase concentrations in blood as indicators of infarction volume and prognosis in acute ischemic stroke. Stroke 1997; 28:1956-60.

11. Herrmann M, Vos P, Wunderlich MT, de Bruijn CH, Lamers KJ. Release of glial tissue-specific proteins after acute stroke: a comparative analysis of serum concentrations of protein S-100B and glial fibrillary acidic protein. Stroke 2000; 31:2670-7.

12. Wunderlich MT, Wallesch CW, Goertler M. Release of neurobiochemical markers of brain damage is related to the neurovascular status on admission and the site of arterial occlusion in acute ischemic stroke. J Neurol Sci 2004; 227:49-53.

13. Moore BW. A soluble protein characteristic of the nervous system. Biochem Biophys Res Commun 1965; 19:739-44.

14. Kapural M, Krizanac-Bengez Lj, Barnett G, et al. Serum S-100beta as a possible marker of blood-brain barrier disruption. Brain Res 2002; 940:102-4.

15. Abraha HD, Butterworth RJ, Bath PM, et al. Serum S-100 protein, relationship to clinical outcome in acute stroke. Ann Clin Biochem 1997; 34 (Pt 5):546-50.

16. Foerch C, Singer OC, Neumann-Haefelin T, et al. Evaluation of serum S100B as a surrogate marker for long-term outcome and infarct volume in acute middle cerebral artery infarction. Arch Neurol 2005; 62:1130-4.

17. Dassan P, Keir G, Brown MM. Criteria for a clinically informative serum biomarker in acute ischaemic stroke: a review of S100B. Cerebrovasc Dis 2009; 27:295-302.

18. Foerch C, Otto B, Singer OC, et al. Serum S100B predicts a malignant course of infarction in patients with acute middle cerebral artery occlusion. Stroke 2004; 35:2160-4

19. Garcia JH, Liu KF, Yoshida Y, Chen S, Lian J. Brain microvessels: factors altering their patency after the occlusion of a middle cerebral artery (Wistar rat). Am J Pathol 1994; 145:728-40.

20. Foerch C, du Mesnil de Rochemont R, Singer O, et al. S100B as a surrogate marker for successful clot lysis in hyper acute middle cerebral artery occlusion. J Neurol Neurosurg Psychiatry 2003; 74:322-5. 